\title{
L'UTILISATION DES PLATES-FORMES AUTOELEVATRICES EN SITE AQUATIQUE
}

\author{
C. CHOBLET - P. FATTAL \\ SEERS - 24 rue du Chêne Lassé - BP 24 - 4480I SAINT HERBLAIN CEDEX
}

\begin{abstract}
The Jack-up is a powerful tool facility equipment allowing all interventions in aquatics environment with a fixed working surface by weak water. Its field of application is very large. It is not in competition with hulks and ships but complements these equipments.
\end{abstract}

\section{INTRODUCTION}

En 1974 la Société S.E.E.R.S. construit sa première plate-forme autoélévatrice destinée à répondre à un marché dans le domaine des investigations géotechniques côtières.

17 années d'utilisation intensive nous permettent de considérer. l'ensemble des inconvénients et avantages que procure un tel support.

La première partie de l'exposé est consacrée à une présentation simplifiée de la plate-forme avec analyse des inconvénients et avantages. La seconde partie, est un exemple de reconnaissance sur site ou rious étudions les étapes successives d'une campagne, et les adaptations aux contraintes rencontrées. Enfin nous évoquerons, dans un troisième chapitre, un éventail non restrictif d'applications différentes de la plate-forme.

\section{PRESENTATION DES PLATES-FORMES AUTOELEVATRICES}

\subsection{Définition}

Une plate-forme autoélévatrice de type côtière est un ponton flottant qui possède des béquilles permettant par différents systèmes de s'élever audessus du niveau de leau. (Fig.1). 


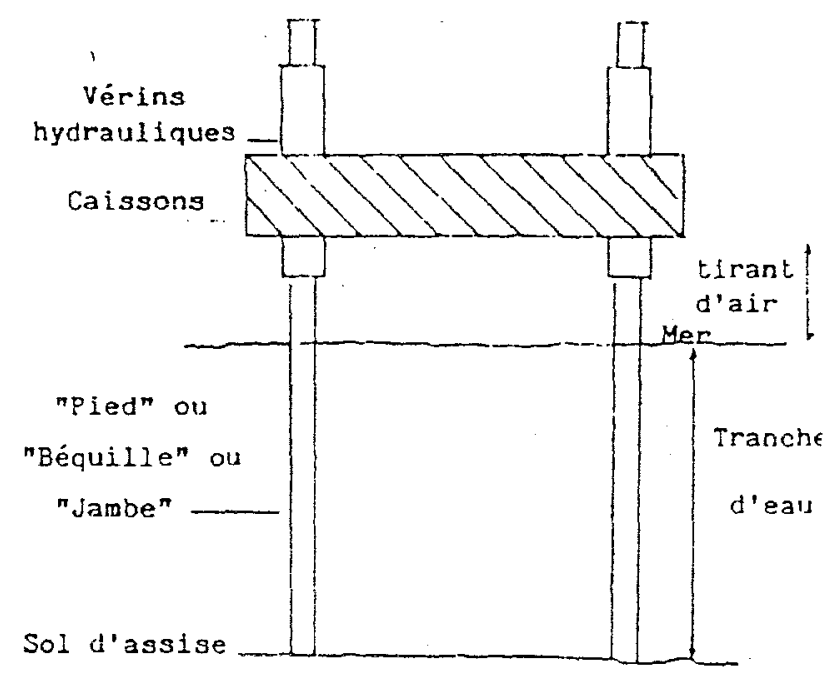

Fig. 1 : Schérna de plate-forme

Il existe différents types de plates-formes. Toutefois à l'usage il s'avère qu'elles ont presque toutes les mèmes avantages et inconvénients.

\section{2. Avantages et inconvénients}

Nous pouvons dresser une liste des caractères communs.

\section{2.1. Avantages}

Un plan de travail fixe et de ce fait :

a) une qualité de travail comparable à une intervention à terre notamment en ce qui concerne les prélèvements d'échantillons intacts et tous les essciis "in-situ".

b) Un positionnement en $X, Y, Z$ précis

Une moindre gêne pour la navigation car, à contrario des pontons, il est ici inutile de tirer des ancres sur de grandes distances.

Des temps d'immobilisations moindres que pour un matériel flottant. La seule immobilisation sur un point ne peut provenir que de l'impossibilité d'embarquer sur la plate-forme en raison d'une mer trop forte lorsque celle-ci est en position haute.

Une plus grande sécurité lorsque l'assise des pieds est bonne et que le tirant d'air est suffisant. En effet, on s'affranchit de nombreuses contraintes liées à la mer (houle, marée, courants,... .). 


\subsubsection{Inconvénients}

Un coût d'installation et de repli plus élevé que pour un ponton flottant de taille égale. En effet, l'acheminement, les temps de mise en place des pieds, le montage et les manipulations entraînent des surcoûts de l'ordre de 2 à 10 fois supérieurs à des pontons.

Une fragilité relative due à sa structure: tout choc sur les béquilles peut compromettre la stabilité de la plate-forme.

Une dépendance du sol d'assise. En effet, nous avons en début de mission l'obligation de connaitre la nature et la géométrie du terrain ceci détermine la stabilité et la portance de la plate-forme. En présence de terrains très défavorables (vase molle avec risque d'enfoncement sur plusieurs mètres) des adaptations peuvent être faites sur l'embase.

La plate-forme est opérationnelle dans une tranche d'eau relativement faible ( 15 à $20 \mathrm{~m}$ maximum pour des plate-formes ayant des béquilles de 30 mètres de long).

Ainsi, compte-tenu de ces avantages et inconvénients, nous pouvons déduire que la plate-forme autoélévatrice et le ponton flottant possèdent chacun leur domaine d'investigation privilégié.

A cet effet, le tableau ci-après indique quelques recommandations en fonction des différentes conditions d'utilisation:

\begin{tabular}{|l|c|c|}
\cline { 2 - 3 } \multicolumn{1}{c|}{} & \multicolumn{2}{c|}{ UTILISATION RECOMMANDEE } \\
\cline { 2 - 3 } \multicolumn{1}{c|}{} & $\begin{array}{c}\text { Plate-forme } \\
\text { autoélévatrice }\end{array}$ & $\begin{array}{c}\text { Ponton } \\
\text { flottant }\end{array}$ \\
\hline Marnage & OUl & NON \\
\hline Agitation du plan d'eau & OUl & NON \\
\hline Trafic maritime à proximité & OUl & NON \\
\hline Nécessité de réalisation d'essais très précis & OU! & NON \\
\hline Tranche d'eau supérieure à 20m & NON & OUI* \\
\hline Budget faible & NON & OUl \\
\hline Sol d'assise très mou ou accidenté & NON & OUl \\
\hline
\end{tabular}

* Au-delà de $20 \mathrm{~m}$, la solution par bateau peut être mieux adaptée. 
Le Port de Nice fournit une bonne illustration du déroulement d'une campagne et des adaptations inhérentes aux conditions rencontrées.

\section{UN EXEMPLE D'INTERVENTION AVEC PLATE-FORME AUTOELEVATRICE EN GEOTECHNIQUE}

Cette campagne de reconnaissances en vue de l'extension du Port de Commerce de Nice s'est déroulée sur une période allant du 15/11/90 au $12 / 03 / 91$.

\subsection{Le projet}

Dans le cadre des études préliminaires de l'extension éventuelle du Port de Nice et de la construction d'une nouvelle jetée au sud de la digue existante. la Chambre de Commerce et d'Industrie décide de lancer une campagne de sondages en mer afin de vérifier la nature des terrains de fondation au droit du futur ouvrage.

\subsection{Le programme}

Le programme établi prévoyait une intervention de Décembre 90 à Février 1991 et comprenait :

- 3 sondages carottés en continu avec prélèvements d'échantillons intacts sous gaine plastique, paraffinés et cela jusqu'aux profondeurs suivantes:

$$
\mathrm{C}_{7}: 25 \mathrm{~m} \quad \mathrm{C}_{2}: 45 \mathrm{~m} \quad \mathrm{C}_{3}: 50 \mathrm{~m}
$$

- 3 forages destructifs avec essais pressiométriques tous les mètres permettant de se représenter le comportement en place du matériau. Ces essais de type haute pression ont été réalisés jusqu'aux profonfeurs suivantes:

$$
\mathrm{SP}_{1}: 40 \mathrm{~m} \quad \mathrm{SP}_{2}: 50 \mathrm{~m} \quad \mathrm{SP}_{3}: 50 \mathrm{~m}
$$

- un ensemble d'essais de laboratoire:

- Analyses granulométriques.

- Cisaillement à la boite.

- Résistance à la compression simple. 


\subsection{Les études préliminaires: les hypothèses de départ}

Le cahier des clauses techniques particulières du marché (C.C.T.P.) donne les indications suivantes:

* Nature des terrains:

- Des matériaux fins de type vasard sur quelques décimètres. puis des formations superficielles composées principalement de sables et galets.

- Un substratum constitué de calcaire blanc, dolomitique fissuré.

* Hauteur d'eau ef épaisseurs minimales des formations superficielles:

Les études bathymétriques et sismiques ont fourni les résultats suivants: (voir tableau suivant)

\begin{tabular}{|c|c|c|c|}
\hline $\begin{array}{c}\text { Désignation } \\
\text { P: pressio } \\
\text { C: carotté }\end{array}$ & Hauteur d'eau & $\begin{array}{c}\text { Profondeur à } \\
\text { forer/ } \\
\text { terain naturel }\end{array}$ & $\begin{array}{c}\text { Epaisseur } \\
\text { minimale des formations } \\
\text { superficielles }\end{array}$ \\
\hline C1 & $16 \mathrm{~m}$ & $25 \mathrm{~m}$ & $7 \mathrm{~m}$ \\
\hline P2 & $21 \mathrm{~m}$ & $40 \mathrm{~m}$ & $5 \mathrm{~m}$ \\
\hline C3 & $26 \mathrm{~m}$ & $50 \mathrm{~m}$ & $15 \mathrm{~m}$ \\
\hline P4 & $21 \mathrm{~m}$ & $50 \mathrm{~m}$ & $7 \mathrm{~m}$ \\
\hline C5 & $15 \mathrm{~m}$ & $45 \mathrm{~m}$ & $0 \mathrm{~m}$ \\
\hline P6 & $17 \mathrm{~m}$ & $50 \mathrm{~m}$ & $0 \mathrm{~m}$ \\
\hline
\end{tabular}

Hauteur d'eau et épaisseur minimales des sols de couverture initialement prévues

L'examen des investigations hydrographiques et sismiques nous permet ainsi, de connaitre les hauteurs d'eau, comprises entre - 15 et - 26 mètres et d'éviter un trop grand enfoncement des pieds puisque les formations superficielles reconnues ont une portance correcte. (Matériaux vasards sur quelques décimètres, puis sables et galets).

\subsection{La campagne de reconnaissance géotechnique}

Les difficultés rencontrées, les conséquences et les nouvelles solutions. 


\subsubsection{Le fond est moins porteur et plus profond que prévu}

Après positionnement de la plate-forme et tentative de mise en élévation, il s'avère, que les profondeurs ne sont pas celles annoncées et que l'épaisseur des formations superficielles molles est bien plus importante que celle retenue dans les hypothèses de départ. (Le tableau ci-après montre les décalages existants).

\begin{tabular}{|c|c|c|c|c|}
\hline & CCTP & & CCTP & \\
\hline $\begin{array}{l}\text { Désignation } \\
\text { p: pressio } \\
c: \text { carotté }\end{array}$ & $\begin{array}{c}\text { Hauteur d'eau } \\
\text { prévue }\end{array}$ & $\begin{array}{c}\text { Hauteur d'eau } \\
\text { réelle }\end{array}$ & $\begin{array}{c}\text { Epaisseur } \\
\text { minimale } \\
\text { des formations } \\
\text { superficielles }\end{array}$ & $\begin{array}{l}\text { Epaisseur } \\
\text { réelle des } \\
\text { formations } \\
\text { superficielles }\end{array}$ \\
\hline $\mathrm{Cl}$ & $16 \mathrm{~m}$ & $20 \mathrm{~m}$ & $7 \mathrm{~m}$ & $16.4 \mathrm{~m}$ \\
\hline$P 2$ & $21 \mathrm{~m}$ & $22.3 \mathrm{~m}$ & $5 \mathrm{~m}$ & $20.5 \mathrm{~m}$ \\
\hline $\mathrm{C3}$ & $26 \mathrm{~m}$ & $29 \mathrm{~m}$ & $15 \mathrm{~m}$ & $23.8 \mathrm{~m}$ \\
\hline PA & $21 \mathrm{~m}$ & $23.8 \mathrm{~m}$ & $7 \mathrm{~m}$ & $29.3 \mathrm{~m}$ \\
\hline C5 & $15 \mathrm{~m}$ & $21.9 \mathrm{~m}$ & om & $23.8 \mathrm{~m}$ \\
\hline Po & $17 \mathrm{~m}$ & & om & \\
\hline
\end{tabular}

Ecarts entre les prévisions et les conditions réelles

Conséquence directe pour la plate-forme: les pieds s'enfoncent de plusieurs mètres dans les vases et deviennent trop courts.

Solutions: augmenter la longueur des pieds ou (et) modifier limplantation des sondages.

\section{4. 2. Le fond est accidenté}

Par ailleurs, nous avons constaté la présence de blocs de béton de plusieurs mètres de côté provenant de la digue actuelle ainsi que des "mini canyons" au droit des points de sondages les plus en aval. (voir figure 2).

Du fait de ces difficultés majeures le risque est très grand que la plateforme ne puisse se positionner de manière stable (tassement différentiel, pieds dans le vide....)

La solution que nous avons alors proposée à notre client est d'effectuer un relevé précis du fond par l'intermédiaire de plongeurs, présents à chaque mise en élévation dans le seul but de contrôler l'assise. 
3. 4. 3. Les carottages et essais sont très difficiles à réaliser

Lors des carottages nous avons observé la nature du substratum: une brèche dolimitique très tectonisée avec de très nombreuses failles ouvertes ou fermées. Les failles ouvertes sont le plus souvent remplies de galets.

En pratique, la foration s'est avérée très difficile car les "passes" ont été courtes et les vitesses d'avancement très faibles (parfois 3 mètres/jour pour le carottage alors que dans un terrain facile on peut espérer $15 \mathrm{~m} / \mathrm{jour}$ ).

Afin de pallier aux difficultés nous avons été amenés à télescoper plusieurs colonnes de tubages provisoires afin d'éviter tout éboulement. En conséquence, l'immobilisation sur le même point de sondage s'avère obligatoire tant que l'investigation n'est pas terminée. Ces faits soulignent nettement l'intérêt de la plate-forme comparée à un ponton "mobile" trop sujet aux facteurs exogènes.

\subsubsection{Les conditions météorologiques sont mauvaises}

La campagne initialement prévue du 15/11/90 au 12/02/91 s'est en fait déroulée du 15/11/90 au 15/03/91 soit avec plus d'un mois supplémentaire.

Une succession de tempêtes et un mauvais temps général pendant toute la durée de la mission a considérablement ralenti les opérations.

Lors de la première tempête, annoncée par la météo, nous avions terminé le premier sondage et devions nous acheminer en flottaison sur le second point. L'avis de tempête nous a permis de nous protéger dans la baie de Villefranche.

Lors d'une deuxième tempête, beaucoup plus brutale, nous étions en foration. Nous avons alors positionné la plate-forme le plus haut possible audessus de l'eau sans compromettre la sécurité de cette dernière.

Conclusion sur cette campagne de reconnaissance

Les premières difficultés rencontrées, à savoir les différences entre les hypothèses de départ et la réalité bathymétrique et sédimentologique. ont montré les limites d'utilisation d'une plate-forme autoélévatrice. La mission risquait d'être compromise en raison de la tranche d'eau et de l'enfoncement des béquilles trop important. 
Par contre, les autres difficultés rencontrées (géologiques et météorologiques) ont démontré tout l'intérêł d'une plate-forme autoélévatrice pour une telle campagne.

En effet. le temps nécessaire pour réaliser un sondage étant compris entre une et trois semaines (hors immobilisations) il est indispensable de rester fixe pendant cette période. Or, de novembre 90 à mars 91 les périodes de mauvais temps se sont succédées presque en continu.. . 


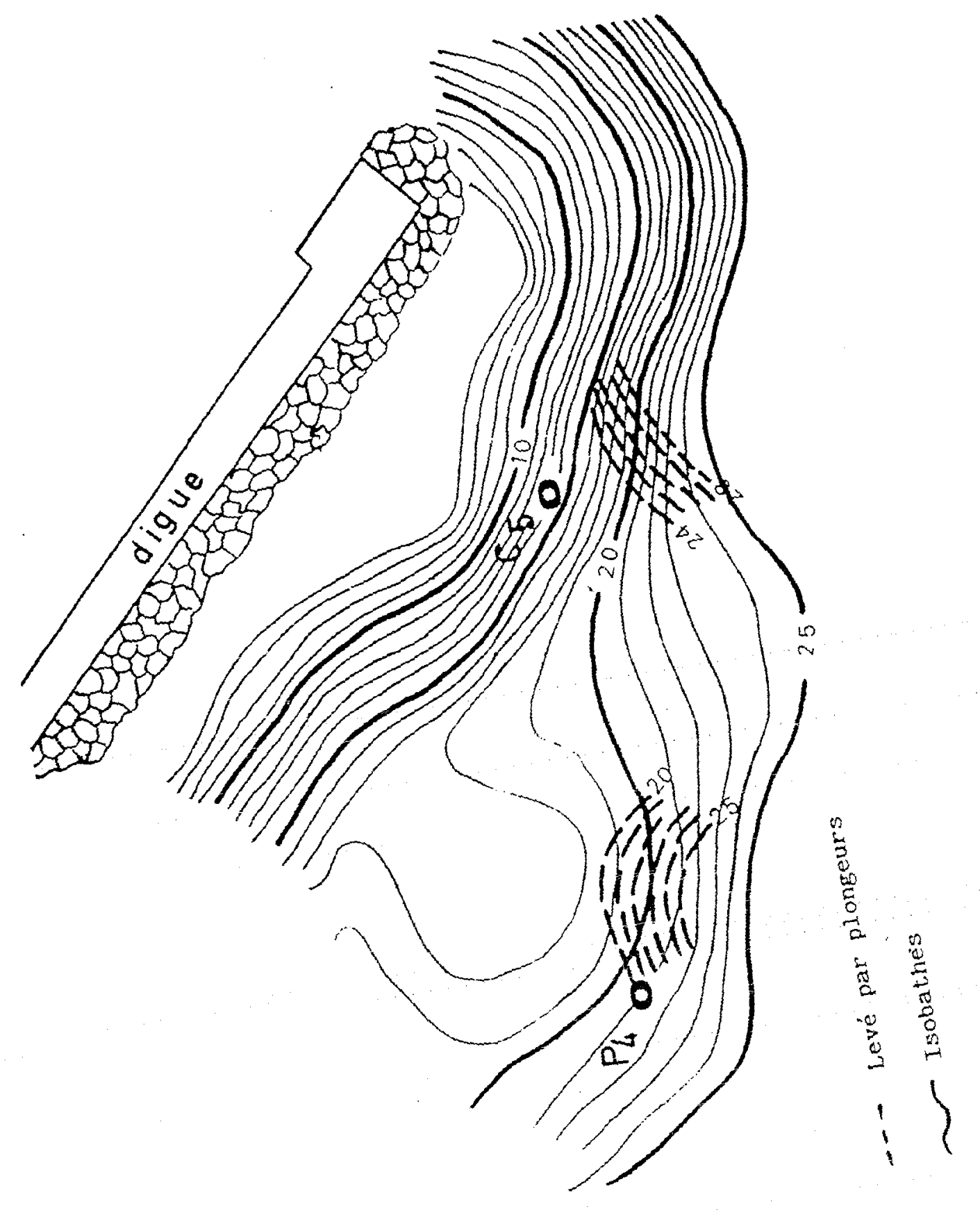

Extrait de la carte bathymétrique 1990 - NICE

Fig. 2

.107. 


\section{DIVERS EXEMPLES D'UTILISATIONS DE PLATE-FORME AUTOELEVATRICE}

\subsection{En travaux Publics}

Les plates-formes autoélévatrices sont souvent utilisées soit pour servir de support fixe à du gros matériel de travaux publics, solt pour des travaux de déroctage. Dans ce dernier cas, les engins de perforation se déplacent sur rails. ce qui permet pour une même mise en station de la plate-forme d'effectuer le maximum de forages dans le rocher.

$$
\begin{aligned}
& a \cdot b=\text { surface de forage. } \\
& x \cdot y=\text { maillage. }
\end{aligned}
$$

nombre de forages admis sur une plate-forme:

$$
n=\frac{a \cdot b}{x \cdot y}
$$

\subsection{En géotechnique}

La précision demandée aux essais font de ce secteur un domaine privilégié d'intervention d'une plate-forme autoélévatrice. Parmi quelques exemples récents d'emploi d'une plate-forme, nous pouvons citer des études préalables à la réalisation:

* des émissaires de rejet de centrale nucléaire:

- Centrales de Braud Saint Louis, de Paluel, de Penly...

* d'ouvrages d'art:

- Pont de l'ile de Ré,

- Pont Albert Louppe à Brest.

* de ports :

- Port de Larnaca (Chypre).

- Port de pẽche de La Pallice.

- Port de Saint-Pierre. (St Pierre et Miquelon) 
* des stations d'épuration en mer:

- Station de Toulon Cap-Sicié.

- Projet de Menton.

\subsection{Divers}

Beaucoup de travaux en mer par faible tranche d'eau peuvent nécessiter I'utilisation de plate-forme autoélévatrice. Le plus souvent celle-ci est un support. comme par exemple pour les travaux de recherche minière en mer sur l'estran de Piriac. Elle peut aussi être exceptionnellement utilisée comme quai (actuellement à Fort Boyard pour réaliser les émissions de jeux télévisés).

\section{CONCLUSION}

La plate-forme autoélévatrice est un matériel très intéressant pour toutes les interventions côtières en faible profondeur qui nécessitent de rester en position fixe pendant une longue durée.

Son utilisation est cependant limitée par des contraintes précises (coût. profondeur d'eau. nature du fond) qu'il faut connaitre pour optimiser le choix entre la plate-forme autoélévatrice et le ponton flottant.

Il ne faut donc pas raisonner en terme de concurrence entre ponton, plateforme ou bateau mais plutôt en terme de complémentarité. 